EUROPEAN COOPERATION

IN THE FIELD OF SCIENTIFIC

AND TECHNICAL RESEARCH
COST $2100 \mathrm{TD}(09) 844$

Valencia, Spain

2009/May/18-19

EURO-COST

SOURCE: CITI Laboratory

University of Lyon, INSA Lyon

France

Candidate Architecture for MIMO LTE-Advanced Receivers with Multiple Channels Capabilities and Reduced Complexity and Cost

Ioan Burciu, Guillaume Villemaud and Jacques Verdier CITI/INSA Lyon

Batiment Claude Chappe

6 , avenue des Arts

69621 Villeurbanne Cedex

France

Phone: (+33) 472437322

Fax: (+33) 472436227

Email: ioan.burciulinsa-lyon.fr 


\title{
CANDIDATE ARCHITECTURE FOR MIMO LTE-ADVANCED RECEIVERS WITH MULTIPLE CHANNELS CAPABILITIES AND REDUCED COMPLEXITY AND COST
}

\author{
I.Burciu, G.Villemaud, and J.Verdier \\ CITI - INRIA \\ University of Lyon, INSA Lyon \\ Lyon, France \\ ioan.burciu@insa-lyon.fr
}

\begin{abstract}
In this paper, a candidate architecture for LTEAdvanced receiver is proposed. Based on the combination of MIMO techniques and flexible spectrum access, LTE-Advanced terminals will require an increasing complexity of the front-end part. To reduce the complexity of the analog front-end, an innovative architecture is introduced based on the merging between the double IQ and code multiplexing structure. Simulation results show that, in a Gaussian case, the bit error rate does not increase significantly when using this architecture. A complexity evaluation study reveals significantly reduced power consumption when implementing the proposed single front-end architecture.
\end{abstract}

\section{INTRODUCTION}

Internet of things: this is the greatest challenge of mobile communications. All incoming standards need to provide higher throughputs in compact terminals. One can see that all proposed solutions converge towards same techniques: OFDM (Orthogonal Frequency Division Multiplexing), MIMO (Multiple Input Multiple Output), adaptive coding and modulation, as well as scalable bandwidths. These techniques require an increasing complexity of terminals, leading to additional cost and consumption. Particularly from the RF front-ends point of view, OFDM imposes high PAPR(Peak to Average Power Ratio) constraints and good linearity [1], MIMO conducts to the multiplication of RF chains, scalable bandwidth imposes wider bandwidth characteristics of RF components [2].

Recently, we have proposed different solutions to reduce complexity of multi-band [3] and multi-antenna [4][5] frontends. In this article we look forward to merging those principles to propose a candidate architecture for simplified LTE-Advanced terminals [6] - current 3GPP [7] working group for future mobile phones with very high data rates and mobility. In fact, in actual propositions for LTE-Advanced PHY-layer, the use of combined multiple-antenna capabilities and multiple separated frequency channels are very seducing for the downlink transmission. Meanwhile the use of these combined techniques supposes high cost architectures for the user embedded terminal.

This paper is organized as follows. Section II summarizes the main LTE-Advanced key points, focusing on multi-band and multi-antenna needs. Section III assesses the previously proposed architectures which separately respond to multi-band and multi-antenna problems respectively. A novel combined architecture is presented in Section IV along with a comparative complexity study. This architecture is dedicated to the multiple-antenna reception of signals composed of two arbitrarily distributed frequency channels. Finally, conclusions and forthcoming works are drawn in section V.

\section{LTE-ADVANCED SPECIFICATION}

\section{A. Requirements}

LTE-Advanced, an evolved version of LTE, is currently under investigation in order to fulfill the requirements defined by the International Telecommunications Union (ITU) for next generation mobile communication systems [6].

Requirements for LTE-Advanced are similar to those imposed by LTE, excepting peak data rates and spectral efficiency which should be increased. The goal is to provide peak-data-rates reaching $1 \mathrm{Gbit} / \mathrm{s}$ in local areas. Such high data rates require a special spectrum allocation for a $100 \mathrm{MHz}$ range in order to obtain a spectral efficiency reaching a 10 bps/Hz level. This spectrum allocation of the $100 \mathrm{MHz}$ system bandwidth will use the aggregation of individual component carriers.

\section{B. Proposed radio access techniques for LTE-Advanced}

The 3GPP working groups are currently starting take into consideration the technical proposals that can be implemented in order to achieve these requirements. The main novel requirements imposed by the future LTE-Advanced concern [7]:

- Asymmetric wider transmission bandwidth

- Layered OFDMA multi-access

- Advanced multi-cell transmission

- Discontinuous spectrum allocations

- Enhanced multi-antenna transmission techniques

In general, OFDM provide simple means to increase bandwidth by simply increasing the number of subcarrier. However, due to a fragmented spectrum, the available bandwidth might not be contiguous, as shown in Fig. 1.

Some of the main challenges for $100 \mathrm{MHz}$ terminals are presented below [6]: 


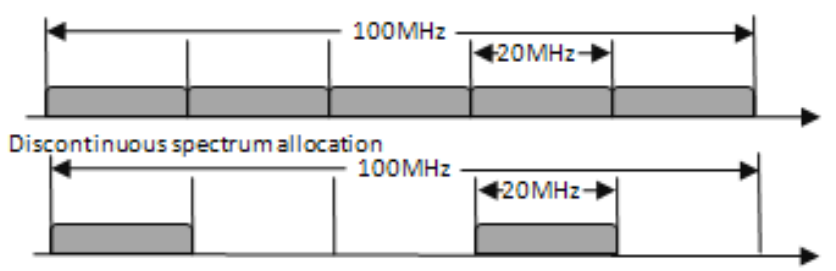

Fig. 1 LTE-Advanced spectrum decomposition and one possible discontinuous spectrum allocation.

- Availability of front-end for such a large bandwidth and bandwidths of variable range

- Availability of Analog Digital Converter with such a high sampling rate and quantization resolution

- Increased decoding complexity e.g. for channel decoding and increased soft buffer size

In this paper, we deal with theses challenges and we propose a candidate architecture for the following scenario.

\section{Scenario}

Among the proposed radio access techniques, multi-antenna transmission and discontinuous spectrum allocation have a direct influence on the front-end part of the receiver. Each additional antenna or each additional band induces an important complexity increase of the receiver's analog part. This study assesses the multi-antenna reception of a discontinuous spectrum signal (as shown in Fig.1).

\section{PRIOR WORKS: LOW COMPLEXITY FRONT-END}

\section{General purpose}

The analog complexity issue concerning advanced receiver using digital processing has been very little addressed [2]. In fact, the performance gain achieved by diversity techniques implies an increase of the digital complexity, but also an increase of the complexity and the consumption of the analog front-end [8].

In the research domain concerning multi-band reception, we can distinguish two different categories of front-ends: nonsimultaneous receivers using switching techniques and simultaneous receiving receivers. To the knowledge of the authors, the optimal scheme when realising multiband simultaneous reception uses the front-end stack-up technique each chain being dedicated to the reception of only one frequency band. Nonetheless, this architecture is characterized by some inconveniences such as the bad complexityperformance trade-off, but also the price and the physical size.

Therefore our studies deal with the analog front-end architectures dedicated to simultaneous multi-band reception. Our main goal is the improvement of the performanceconsumption-complexity trade-off offered by these structures. Two studies explore the use of a single common front-end for the processing of signals received by the antennas:

- The double IQ architecture dedicated to the simultaneous reception of two separate frequency band signals.

- The code multiplexing architecture for the multiantenna reception.
The use of a single analog chain underlies the idea of multiplexing the different RF branches. In other words, we realise a multiplexing of the different RF contributions in order to use a single frequency translation step. In the digital domain, we demultiplex the different baseband translated contributions.

\section{E. Double IQ architecture \\ 1) Context}

Double orthogonal translation techniques are generally used in image frequency rejection front-ends [9]. The rejection of the signal having its spectrum in the image frequency band of the useful signal is a key point of the heterodyne front-end architectures. In order to perform these specifications, the double orthogonal translation technique uses a signal processing method that manages to reconstruct the useful signal starting from four baseband signals. These baseband signals are the result of a double orthogonal frequency translation. Each of them is composed of two separate contributions: the baseband components of the useful signal and of the image frequency band signal.

In other words, the double orthogonal (double IQ) structure is multiplexing the useful signal and the image frequency band signal during the translation from RF to baseband and is then demultiplexing them in the baseband domain using a signal processing technique. Starting from this structure, we proposed and developed a novel front-end architecture dedicated to the simultaneous reception of two separate frequency band signals [3].

\section{2) Presentation of the structure}

The proposed dual-band simultaneous reception architecture is shown in Fig. 2. The input blocks - antenna, RF filter and LNA (Low Noise Amplifier) - are parallelized in order to have a good amplification and filtering of the two useful signals $S_{1}$ and $S_{2}$. In the same time, we concluded to a possible mutualisation of these blocks while realizing the reception of two signals representing two frequency non-overlapping channels of the same standard. Once filtered and amplified, the useful signals are translated in the baseband domain by a double IQ structure. An important aspect that has to be mentioned is the choice of the first local oscillator $\mathrm{LO}_{1}$ frequency. This frequency is chosen in such a manner that each of the useful signals has a spectrum situated in the image frequency band of the other. In the baseband domain, the four obtained signals are digitalized. The baseband component of the two useful signals is obtained by using two dedicated basic operations processing similar with that used by the image frequency rejection structures [9].

3) Characteristics

The double orthogonal translation technique allows a theoretically perfect rejection of the image band signal for a case where the quadrature mounted mixers are perfectly matched - no phase or gain mismatch. In the meantime, design and layout defaults, such as different line lengths between the two branches and non identical gain of the mixers, generate phase and respectively gain mismatches [10]. A study concerning this issue as well as the design and implementation of an original dedicated digital MMSE 


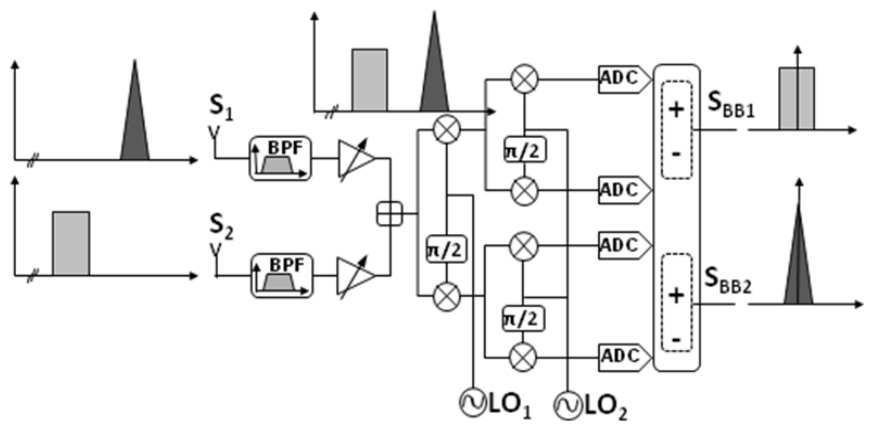

Fig. 2 Double IQ architecture dedicated to the simultaneous reception of two separate frequency band signals.

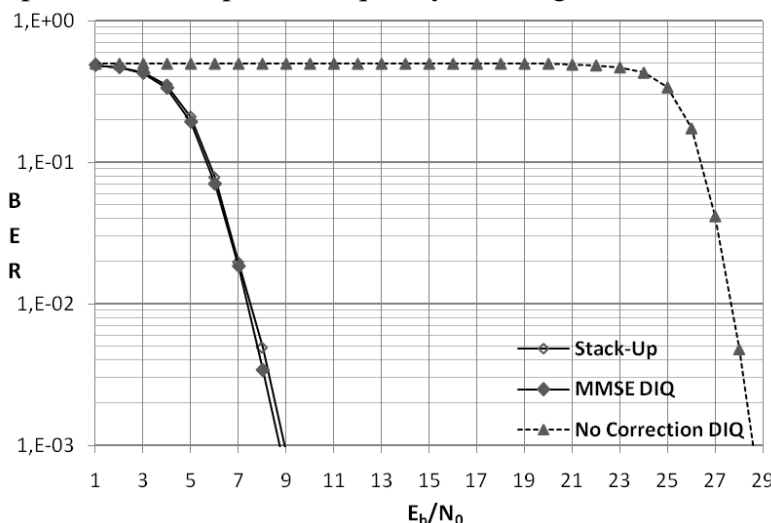

Fig. 3 802.11 BER evolution during a multiband simultaneous reception using the Double IQ architecture and the Stack-up architecture integrating constant orthogonal mismatches.

(Minimum Mean Square Error) method has been done.The results show that, when integrated, this MMSE method confers to the double IQ architecture the same sensitivity to IQ mismatches as that of the dedicated front-ends stack-up architecture. These results were obtained in simulation using the ADS software provided by Agilent Technologies, the input signals chosen are two non-overlapping $802.11 \mathrm{~g}$ channels. These results were validated by using an Agilent Technologies Platform that integrates a real transmission channel and an accurate model of the proposed architecture. Fig. 3 presents these results. The BER evolution depending on the SNR (Signal to Noise Ratio) of the useful signals shows that, when integrating the digital MMSE Method, the double IQ (MMSE DIQ) architecture presents the same sensibility to the IQ defaults as the stack-up architecture (Stack-Up) - for a phase and gain mismatch of 10 and $0.3 \mathrm{~dB}$.

Several studies concerning the proposed double IQ structure were realized. In the following we mention the one concerning the specifications of the ADC (Analog to Digital Converters) and that concerning the overall consumption. The ADC specification study concluded that the constraints imposed to the ADC used by this structure are almost identical (dynamics and sampling frequency) to those used by a dedicated frontend stack-up structure. The overall consumption study is comparing the double IQ structure to the front-end stack-up structure. It concludes that due to the mutualisation of several blocks as well as because of the use of fewer components, the proposed double IQ structure offers

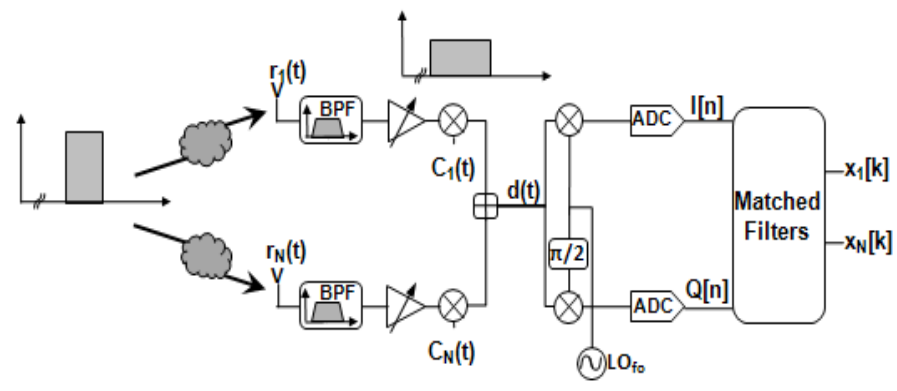

Fig. 4 Code multiplexing architecture dedicated to the simultaneous reception of a multi-antenna system.

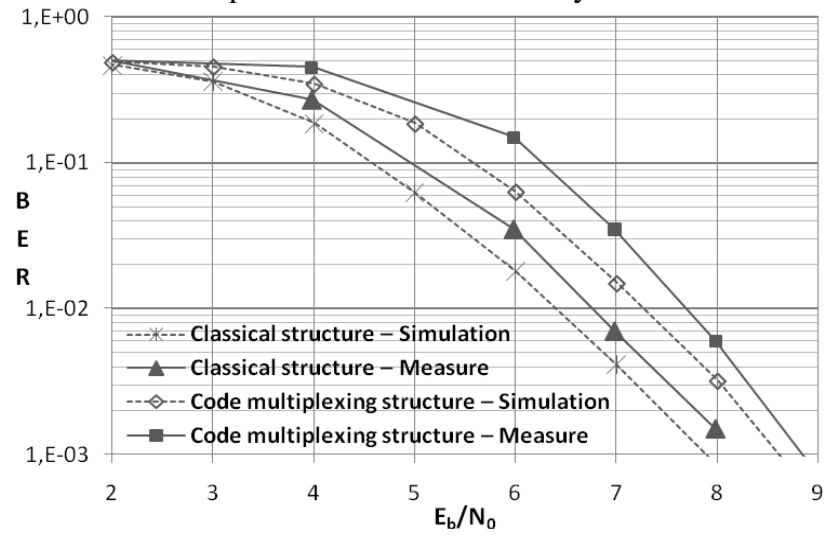

Fig. 5 802.11g simulated and measured BER evolution for a two-antenna SIMO reception using the code multiplexing architecture and the classical stack-up architecture.

a significant $25 \%$ of consumption gain as well as a smaller complexity.

\section{A. Code multiplexing architecture \\ 1) Context}

An antenna diversity system must be able of simultaneously receive different versions of the transmitted signal(s).For example, an $\mathrm{N}$ antennas receiver must simultaneously process $\mathrm{N}$ signals having their spectrums situated in the same frequency band. To answer these specifications, the RF stackup architecture is an obvious choice. It is composed of $\mathrm{N}$ stacked-up processing chains, each of them being dedicated to the demodulation of one of the $\mathrm{N}$ branches. However, this choice imposes a high complexity of the analog part of the receiver. The goal of our study concerning the multipleantenna structures aims to reduce this complexity without decreasing the baseband signal's SNR quality.

To this end, an innovative architecture is introduced based on code multiplexing. This architecture uses the direct sequence spread spectrum (DSSS) technique in order to multiplex the different antennas contributions so that a single frequency translation block is used.

\section{2) Presentation of the structure}

In order to achieve the time and frequency overlapping between each antenna contribution, decorrelation can be done by the DSSS technique. This technique is the basis of CDMA multiple access technology [11]. The DSSS technique consists in allocating an orthogonal spreading code to each branch. 


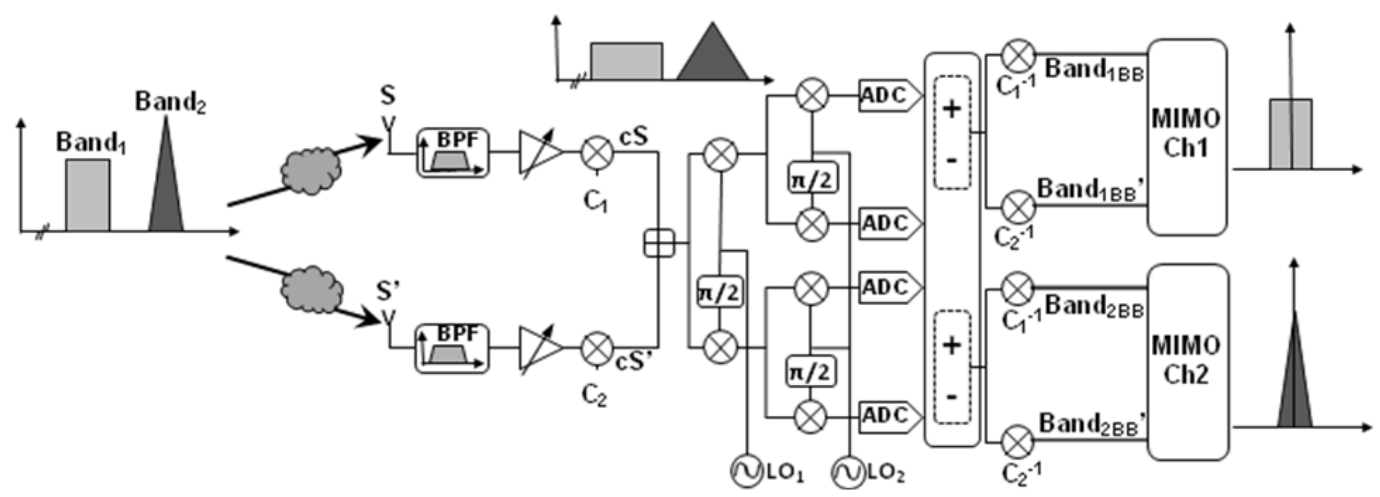

Fig. 6 Candidate Architecture LTE-Advanced Receivers capable of realizing a multi-antennas processing of a discontinuous spectrum signal. The discontinuous spectrum is received, coded in the baseband domain in order to be processed by a single front-end structure.

The signals $\mathrm{rk}(\mathrm{t})$ are multiplied by the periodical codes $\mathrm{ck}(\mathrm{t})$ which are pseudorandom sequences of $\mathrm{N}$ binary entities having a rate $\mathrm{N}$ times higher than the symbol. Thus, the resulting bandwidth of the signal's spectrum is $\mathrm{N}$ times larger than those of the received signal $\mathrm{rk}(\mathrm{t})$. The addition of $\mathrm{N}$ encoded signals is then performed in order to generate the radio-frequency multiplex signal $\mathrm{d}(\mathrm{t})$. This signal is then baseband translated by a unique IQ demodulator. The decoding of each contribution is performed in the digital domain. It consists in using matched filters composed of digital filters followed by subsampling operations.

The proposed code multiplexing architecture is shown in Fig. 4. The RF input blocks (antenna, RF filter and LNA) are parallelized in order to have a good amplification and filtration of the received radiofrequency signals.

\section{3) Characteristics}

The proposed structure uses orthogonal codes to multiplex the different branches through a single IQ demodulator. The main goal was to reduce the complexity of the analog frontend.

In [4], we show that this system reduces the number of ADC by using only two ADC instead of the $2 \mathrm{~N}$ used by the classic receiver. But, for the proposed architecture, the specifications of the ADC in terms of bandwidth are much more stringent. Meanwhile, a global consumption reduction of $20 \%$ could be reached for an $\mathrm{N}=8$ antennas system.

The points revealed in [5] are the evaluation of the feasibility of such a structure. The implementation of analog coding and digital decoding has been validated by BER simulations and measurements (Fig. 5). Results show that, in a Gaussian case, the bit error rate does not significantly increase when the receiver integrates this type of RF coding architecture instead of the classical front-end stack-up.

Several studies have been done concerning the influence of IQ imbalance on the signal quality. Their conclusions reveal that the proposed architecture has the same sensitivity to IQ mismatches as the stack-up architecture. We note that the resulting IQ mismatches affect in the same manner the quality of each antenna baseband translated contributions. We mention that a classical dedicated front-end stack-up will have different IQ impairments for each dedicated IQ demodulator. These theoretical performance were validated in real environment simulations. In [12] we implement a SMI (Sample Matrix Inversion) multi-antenna digital method in order to compare the performance of the RF code multiplexing architecture with those of a classical front-end stack up for different IQ mismatches levels.

The last important point that has to be mentioned is the capability of the proposed architecture to adapt to any multiantenna schemes. In fact, we can find different application scenarios such as MIMO reception or beamforming techniques

\section{LTE-ADVANCED RECEIVER ARCHITECTURE}

\section{B. Context}

As mentioned in section II, the future LTE-Advanced standard takes in consideration a discontinuous spectrum usage, as well as MIMO techniques for the downlink transmission. In order to answer to these specifications the actual state of the art of radiofrequency receivers imposes the use of dedicated front-end stack-up architectures. For example, if we consider a dual-band discontinuous spectrum scenario where two antennas are used for the reception of a MIMO transmission, the receiver will have to integer a four front-end stack-up. Each of these front-ends is dedicated to the processing of one of the contributions obtained from the combination of two antennas and two non-adjacent frequency bands. It becomes obvious that this method, involving the stack-up of dedicated front-ends, imposes high complexity, but especially high power consumption.

One of the most important issues when designing a radiofrequency receiver is the performance-power-complexity trade-off. In order to obtain low power consumption and low complexity LTE-Advanced receiver, an obvious solution is the mutualisation of elements of the dedicated front-end used by the stacked-up architecture. If we also take into consideration the simultaneous processing constrains, we can conclude that the mutualisation of the frequency translation step imposes a multiplexing of the different RF domain contributions.

Starting from this RF multiplexing idea, we present a novel single front-end architecture able to overcome the constraints imposed to the receiver by the LTE-Advanced standard. 


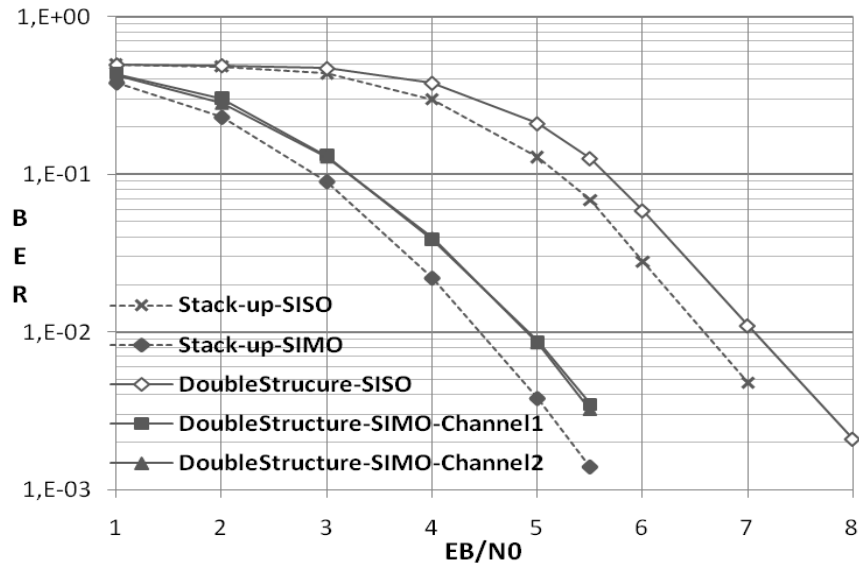

Fig. 7 BER evolution during the simultaneous SIMO reception of a signal composed of two non-overlapping $802.11 \mathrm{~g}$ channels.

\section{Structure presentation}

Starting from the dual band simultaneous reception and the RF code spreading architectures presented in Section III, we propose a unique front-end architecture dedicated to the LTEAdvanced receivers. In this paper we chose to treat the case of a two antenna reception an OFDM dual band signal, corresponding to a handheld integration scenario. Meanwhile, the structure can be easily generalized for several antennas by increasing the number of chips used by the orthogonal codes.

In the followings, we consider that the two input signals $S$ and $S^{\prime}$ are the result of the passage of a bi-band signal through two different transmission channels. Let $\mathrm{Band}_{1}-\mathrm{Band}_{2}$ and Band $_{1}$ '-Band 2 ' be the two pairs of contribution composing $\mathrm{S}$ and respectively $S^{\prime}$. Once received by two space separated antennas, the two input signals are separately filtered and amplified by two RF filters and two LNA respectively, as shown in Fig 6.

The multiplexing of the four contributions is realized by a two step method. To begin, we use the orthogonal code spreading technique in order to multiplex the two input signal once filtered and amplified. Let $\mathrm{cS}, \mathrm{cS}{ }^{\prime}, \mathrm{cBand}_{1}-\mathrm{cBand}_{2}$ and cBand $_{1}$ '-cBand ${ }_{2}$ ' be the signals resulting from the use of the coding technique.

The orthogonal codes used here have a length of two and a chip time two times smaller than the symbol time of each of the two signals. When multiplied with the orthogonal codes, each of the $S_{1}$ and $S_{2}$ signals will see each of the components of the pairs Band $_{1}-$ Band $_{2}$ and respectively Band $_{1}$ '- Band $_{2}$ ' spread in the same manner around their own central frequency, as shown in Fig. 6. This multiplexation step is concluded by the addition of the $\mathrm{cS}_{1}$ and $\mathrm{cS}_{2}$ signals. Therefore, by using the code spreading technique, we multiplexed two by two the contributions having the same central frequency but which have been received on different antennas. As a result of this operation we obtain a signal having a dual band spectrum. Each of its two frequency bands is composed of the addition between $\mathrm{cBand}_{1}$ and $\mathrm{cBand}_{1}{ }^{\prime}$ and respectively $\mathrm{cBand}_{2}$ and cBand ${ }_{2}$.

The second block of the architecture assessed in this section is implementing the double IQ technique similar to that used

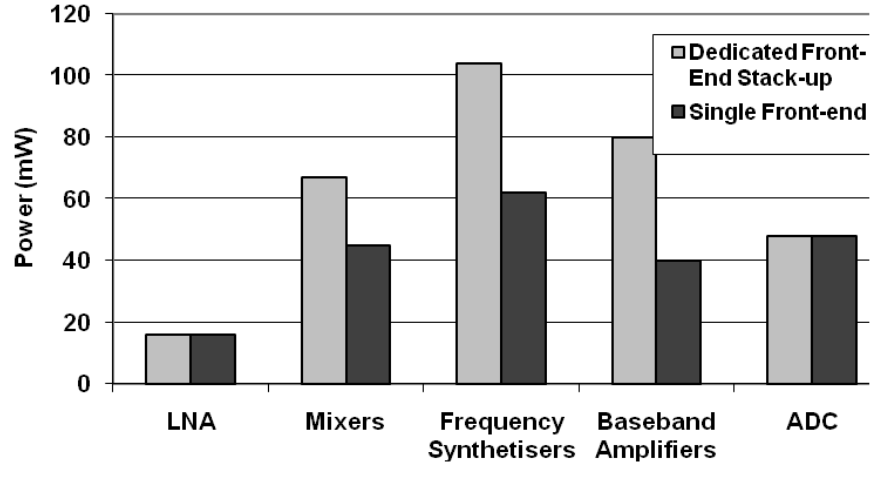

Fig. 8 Consumption of the different types of basic blocks used by the single front-end architecture and the dedicated front-end stack-up.

by the dual band simultaneous reception structure. The processing realized by this block realizes a frequency translation of the RF orthogonal coding block output in the baseband domain.

Similar to the structure presented in Section III the two digital outputs are the baseband translation of the two frequency bands composing the signal at the output of the RF coding block. Therefore, each of them is the addition between the baseband translated signals $\mathrm{cBand}_{1}, \mathrm{cBand}_{1}{ }^{\prime}$ and respectively $\mathrm{cBand}_{2}, \mathrm{cBand}_{2}$ '. In order to demultiplex each of these two pairs of signals, we apply two matched filters, similar to those used for the code multiplexing architecture.

Once we separately obtain the two pairs of signals corresponding to the two antennas reception of a dual band signal, two antenna SIMO processing method are used. Each of them has one of the pairs of decoded signals as input.

\section{Characteristics}

The single front-end receiver presented above is capable of answering to the LTE-Advanced requirements concerning the MIMO reception of a discontinuous spectrum signal.

In order to validate the theoretical study relative to this receiver we realized several simulations. The software used for these simulations was the ADS software provided by Agilent Technologies.

Knowing that the physical layer requirements concerning LTE-Advanced are not yet finalized, we choose to use a particular RF signal model for these simulations. In order to obtain an OFDM signal having a discontinuous spectrum we use a signal composed of the addition of two 802.11g nonoverlapping channels. For the transmission channel the model chosen is the AWGN (Additive White Gaussian Noise).

The results presented in Fig.7 show the BER evolution for different $E_{b} / N_{0}$ of the input signal. The receivers are using either the proposed unique front-end architecture or the dedicated front -end stack-up architecture. The results showed in Fig. 7 are mainly concerning the SIMO receptors using the two different architectures when receiving the dual-channel signal -Stack-up-SIMO, DoubleStructure-SIMO-Channel1 and DoubleStructure-SIMO-Channel2. We also show the BER evolution when the receivers are realizing a SISO receptionStack-up-SISO and DoubleStructure-SISO. Both in the SIMO 
and SISO case, the reception performance when using the front-end stack-up architecture are slightly better compared to those obtained when using the unique front-end structure. This is due to the fact that the codes used by the Double Structure are not completely orthogonal. This matter has been already treated in Section III. We observe that the SIMO reception offers the same performance gain when using the two architectures. Meanwhile, if a real SIMO AWGN channel we have a $3 \mathrm{~dB}$ gain, the simulated channel manages to offer only a quasi AWGN conditions. In fact, the two noises added to the initial signal are not completely uncorrelated. This is why the SIMO gain obtained during ADS simulation doesn't reach the $3 \mathrm{~dB}$ level. In conclusion we consider that the performance of the proposed architecture are the same as those of the dedicated front-end stack-up.

Parallel to this performance study, a comparative power consumption study was made. For this study we considered that the single front-end architecture, as well as the dedicated front-end stack-up imposes the same constrains to the basic blocks (LNA, filters, amplifiers, mixers and oscillators) that are composing them. A bibliography research allowed us to choose the state of the art of these basic components in terms of performance-power consumption trade-off. The conclusions of this study show a $33 \%$ power consumption gain in favor of the single front-end architecture $211 \mathrm{~mW}$ instead of 315 $\mathrm{mW})$. The main reason to this important gain is due to the use of less basic blocks. In order to better illustrate this idea, Fig. 8 shows the consumption of the different types of basic blocks used by the single front-end architecture and the front-end stack-up. If we also take into account the fact that the complexity of the proposed structure is significantly smaller fewer components and image frequency filter free-, it becomes obvious that the proposed structure offers an excellent performance-consumption-complexity trade-off.

\section{CONCLUSION}

In this article, a novel low complexity architecture was presented. We consider that this type of architecture is a good candidate to the integration in the receivers dedicated to the future LTE-Advanced standard. One of the major advantages of this architecture is its low complexity and therefore low power consumption.

Knowing that the power consumption of the analog part of the receiver is directly dependent on the used number of basic processing blocks, the excellent complexity-powerperformance trade-off becomes obvious. Indeed, this is confirmed by the fact that, despite the use of a demultiplex block in the digital domain, the proposed architecture offers a significant $33 \%$ of power gain compared with the solution proposed by the actual state of the art.

The proposed architecture joins two separate innovating methods: the RF orthogonal coding and the double IQ dual band simultaneous reception technique. Expected performance of its implementation has been presented for a particular study case - SIMO reception of an OFDM discontinuous spectrum signal. The simulation results show similar performance when comparing the proposed unique front-end structure and the dedicated front-end stack-up. This validates our theoretical study.

As mentioned in section two, one of the sensible points of this structure is the impact that the IQ mismatches can have on the signal quality. One possible solution to this inconvenient is the use of a digital MMSE method in order to mitigate the IQ mismatch influence on the received signal. This represents one of the forthcoming of our activities.

Another issue that still has to be addressed aims the design and realization of a demonstrator. Real environment measurements concerning the single front-end architecture will soon be realized using a radiofrequency platform with a real SIMO transmission channel.

\section{ACKNOWLEDGEMENT}

The authors wish to sincerely thank Orange Labs which supports this work.

\section{REFERENCES}

[1] R. Van Nee and R. Prasad, OFDM for Wireless Multimedia Communications, Artech House, 2000.

[2] T. Kaiser et al., "Smart Antennas: State of the Art", Eurasip Book Series on Signal Processing \& Communications, 2005.

[3] I.Burciu, M. Gautier, G. Villemaud and J.Verdier, "A 802.11g and UMTS Simultaneous Reception Front-End Architecture using a double IQ structure", IEEE Vehicular Technology Conference (VTC09-Spring), Barcelona, Spain, 26-29 april 2009.

[4] M. Gautier and G. Villemaud, "Low complexity antenna diversity front-end: Use of code multiplexing", IEEE Wireless Communication and Networking Conference (WCNC09), April 2009.

[5] M. Gautier, I. Burciu and G. Villemaud, "New antenna diversity frontend using code multiplexing", European Conference on Antennas and Propagation (EUCAP 09), Berlin, Germany, 23-27 march 2009.

[6] Eiko Seidel. "Progress on LTE Advanced" - the new 4G standard". White Paper, Nomor Research GmbH., July 2008.

[7] www.3gpp.org

[8] H. Tsurumi and Y. Suzuki, "Broadband RF stage architecture for software-defined radio inhandheld terminal applications," IEEE Communications Magazine, vol. 37, no. 2, pp. 90-95, February 1999.

[9] J. C. Rudell, J.-J. Ou, T. B. Cho, G. Chien, F. Brianti, J. A. Weldon, and P. R. Gray, "A 1.9GHz Wide-Band IF Double Conversion CMOS Integrated Receiver for Cordless Telephone Applications", IEEE Journal of Solid-State Circuits, vol. 32, no. 12, pp. 2071-2088, Dec. 1997.

[10] S. Traverso, M. Ariaudo, I. Fijalkow, J-L Gautier and C. Lereau, "Decision Directed Channel Estimation and High I/Q Imbalance Compensation in OFDM Receivers", IEEE Transactions on Communications, Feb. 2008.

[11] R. Kohno, R. Meidan, and L.B. Milstein, "Spread spectrum access methods for wireless communications," IEEE Communications Magazine, vol. 33, no. 1, pp. 58-67, Janvier 1995.

[12] M. Gautier, P.F. Morlat and G. Villemaud," "IQ imbalance reduction in a SMI multi-antenna receiver by using a code multiplexing front-end", IEEE Vehicular Technology Conference (VTC09-Spring), Barcelona, Spain, 26-29 april 2009. 